\title{
Protein chips compatible with MALDI mass spectrometry prepared by ambient ion landing
}

\author{
Petr Pompach ${ }^{\dagger, \ddagger, \#}$, Oldřich Benada ${ }^{\dagger, \dagger}$, Michal Rosůlek $^{\dagger, \ddagger}$, Petra Darebná ${ }^{\dagger, \ddagger}$, Jiří Hausner $^{\dagger, \ddagger}$, Viktor Růžička ${ }^{\S}$, \\ Michael Volný, ${ }^{*}, \#$ and Petr Novák ${ }^{*,+,+, \#}$ \\ ${ }^{\dagger}$ Institute of Microbiology, v.v.i., Czech Academy of Sciences, Prague, Czech Republic; ${ }^{\ddagger}$ Faculty of Science, Charles \\ University in Prague, Prague, Czech Republic; 'Faculty of Science, J. E. Purkyně University in Ustí nad Labem, Ústí \\ nad Labem, Czech Republic; ${ }^{\# A f f i P r o, ~ s . r . o ., ~ M r a t i n, ~ C z e c h ~ R e p u b l i c ; ~}{ }^{\circledR}$ BioVendor, a.s., Brno, Czech Republic. \\ KEYWORDS: Ambient ion landing, protein (arrays) chips, proteases, lectins, antibodies, affinity enrichment, mass \\ spectrometry.
}

${ }^{+}$Corresponding authors: pnovak@biomed.cas.cz and mvolny@gmail.com

\section{SUPPLEMENTARY CONTROL EXPERIMENTS}

Dropping proteins on the surface. One microliter of trypsin re-suspended in $20 \mathrm{mM}$ ammonium bicarbonate buffer, pH 7.8 at 10 $\mu \mathrm{M}$ concentration (equivalent of sprayed amount) was dropped on the ITO glass and let dried at RT. Surface was washed for 5 minutes with water. Similarly, one microliter of pepsin $(50 \mu \mathrm{M})$ re-suspended in $20 \mathrm{mM}$ ammonium acetate, $\mathrm{pH} 4.0$, concanavalin A $(50 \mu \mathrm{M})$ re-suspended in $20 \mathrm{mM}$ ammonium bicarbonate $\mathrm{pH} 7.8,10 \%$ acetonitrile and leptin antibody (10 $\mu \mathrm{M})$ re-suspended in 20 mM ammonium bicarbonate buffer, pH 7.8 were dropped on the ITO glass and let dried at RT. The application of the samples on the chip and further procedures were same as described in Experimental section.

Determination of binding capacity. The ITO glass was modified with different amount ( $80 \mathrm{fmol}, 400 \mathrm{fmol}, 2 \mathrm{pmol}, 10 \mathrm{pmol})$ of leptin antibody using the ambient ion landing procedure as described in the Experimental section. Leptin spiked (250fmol/ $\mu \mathrm{l})$ serum was incubated on each spot of immobilized antibody and chips were further processed and analyzed as described in the text.

\section{SUPPLEMENTARY RESULTS AND DISCUSSIONS}

In the first control experiment the proteins used in this study were dropped in solution on MALDI targets using a pipette, allowed to dry, washed and respective enrichment or proteolytic experiments were performed. Results are summarized in table format in Table S1 and show that only the MALDI plates with solution-deposited trypsin demonstrated protein activity. Other proteins that were dropped in solution on MALDI plates and dried were mostly washed away from the MALDI plates and the plates then showed no or inferior activity compare to MALDI plates modified by electrospray deposition technique. This experiment demonstrates the advantage of the presented modification technique where the proteins are first ionized, desolvated and accelerated from the gasphase towards the surface being modified. The second control experiment was focused on determination of nonspecific binding capacity of the MALDI surfaces. Increasing amounts of antibody were landed on the surface and constant amount of antigen was added for immunoenrichment. As can be seen in the new Figure S4, the signal intensity of captured, enriched and ionized antigen depended on the antibody amount. The minimal amount of antibody that needs to be landed on the surface was estimated as $80 \mathrm{fmols}$. This shows that the surface capacity to bind and enrich the antigen depends on the amount of landed antibody (as a "stationary phase"), which is expected, and the nonspecific capacity of the bare surface to bind the antigen is minimal. 


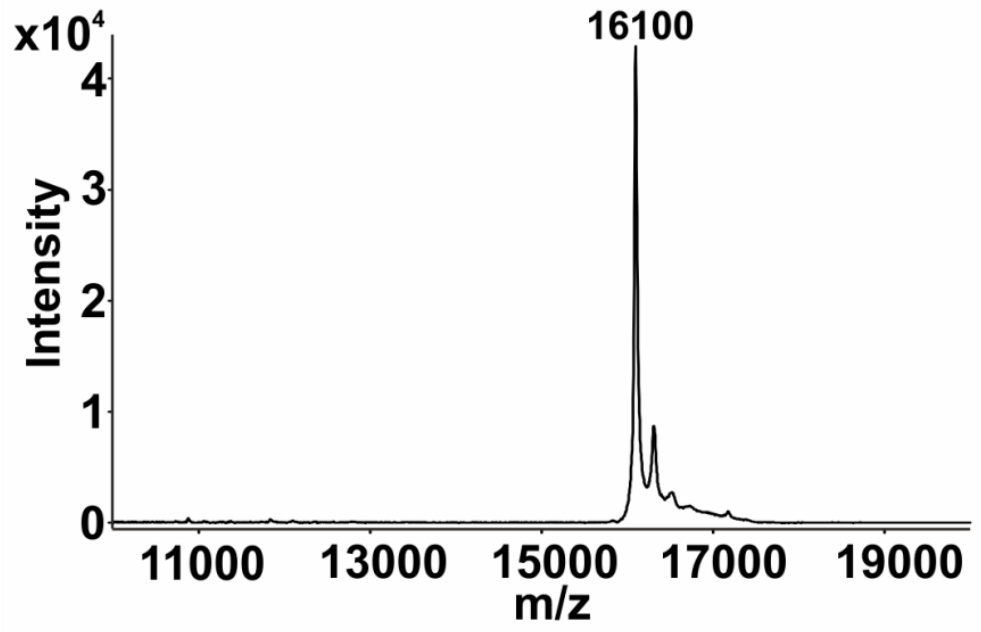

Supplemental Figure 1. MALDI-TOF spectrum of leptin standard from pure solution

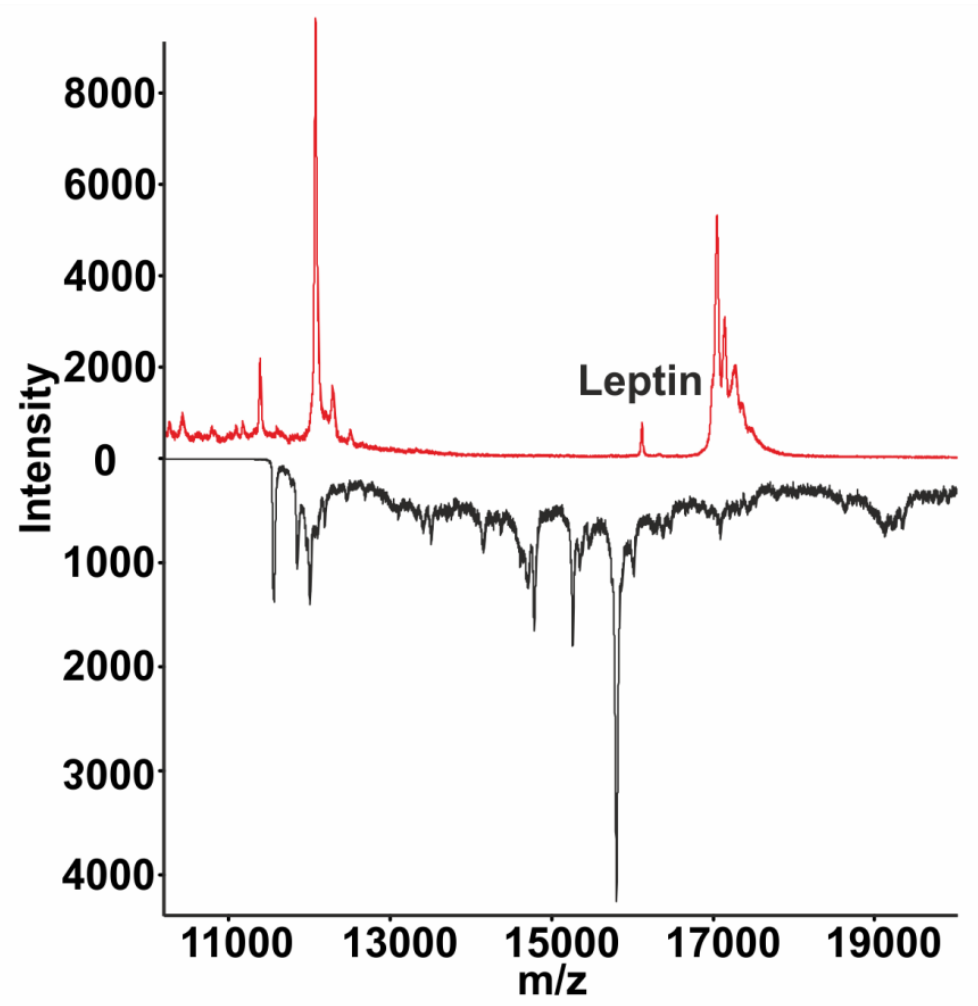

Supplemental Figure 2. MALDI-TOF spectrum of leptin-spiked serum enriched on MALDI chip functionalized by polyclonal human anti-leptin antibody (red spectrum). The leptin peak is small but well-resolved at $\mathrm{m} / \mathrm{z}=16100$. The flipped black spectrum shows analysis of the same sample without any enrichment (no leptin peak at $\mathrm{m} / \mathrm{z}=16100$ was detected due to sample matrix interference). 


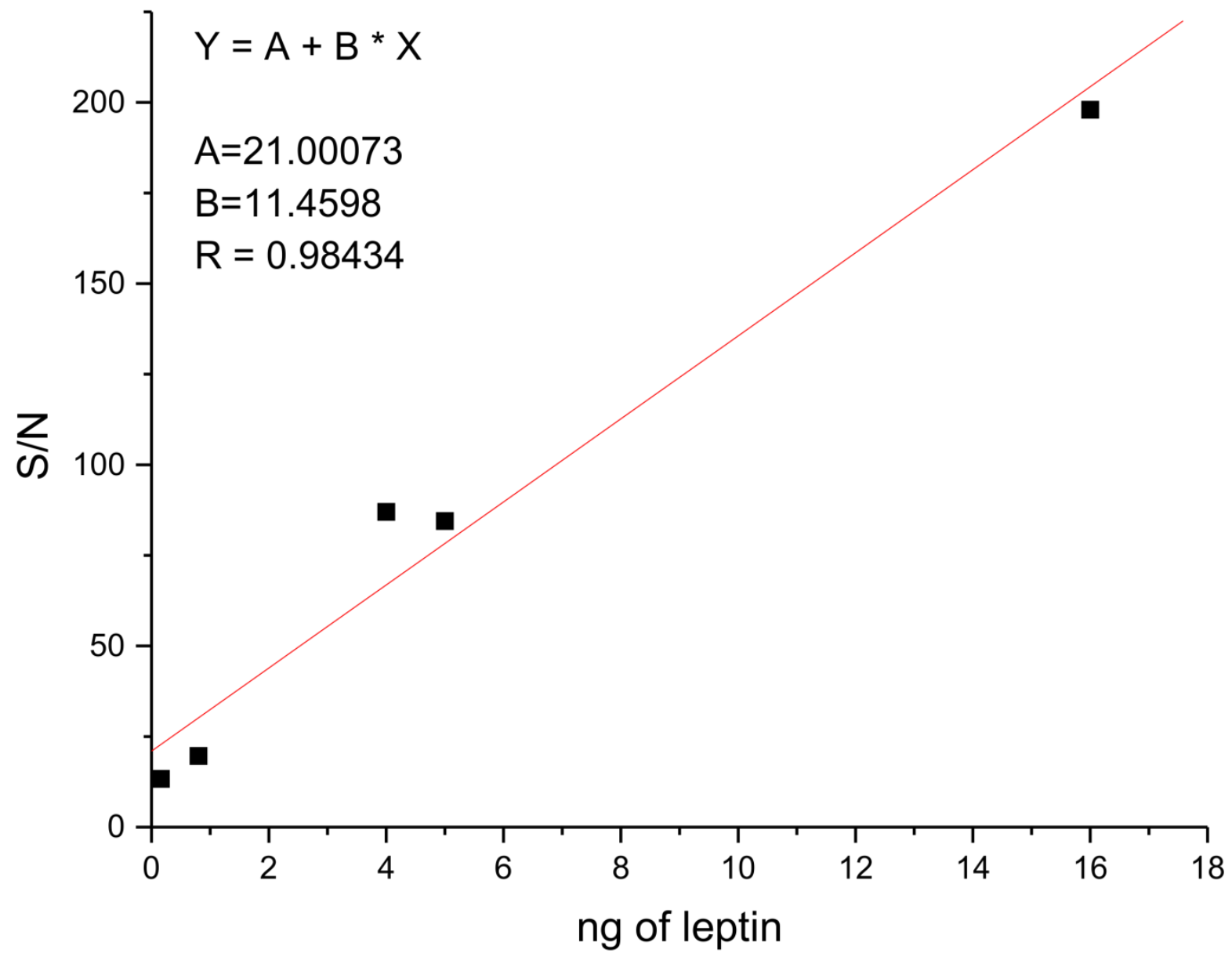

Supplemental Figure 3. The linear range of the immunoMALDI calibration dependency experiment from Figure 4A. The linearity exists only between 10-1000 fmol (0.16-16 ng). X-coordinate shows ng of sampled leptin and y-coordinate signal-to-noise ratio of leptin peak as calculated by FlexAnalysis software (Regression analysis: $y=21.0+11.5 x ; R=0.98$ ). 


\begin{tabular}{|c|c|}
\hline Protein & ITO plate \\
\hline Trypsin & Adhesion to the surface comparable to ion landing deposition \\
\hline Pepsin & Low adhesion to the surface \\
\hline Antibody (anti-leptin) & No adhesion to the surface \\
\hline Concanavalin A & Low adhesion to the surface \\
\hline
\end{tabular}

Supplemental Table 1. The dropped proteases, antibody and lectin on the ITO glass surface by pipetting. The highest activity of the dropped proteins was observed in trypsin, which immobilized on the surface. Leptin antibody was not retained on the surface after washing. ITO plates with dropped pepsin and Concavalin A showed only limited protein activity. 


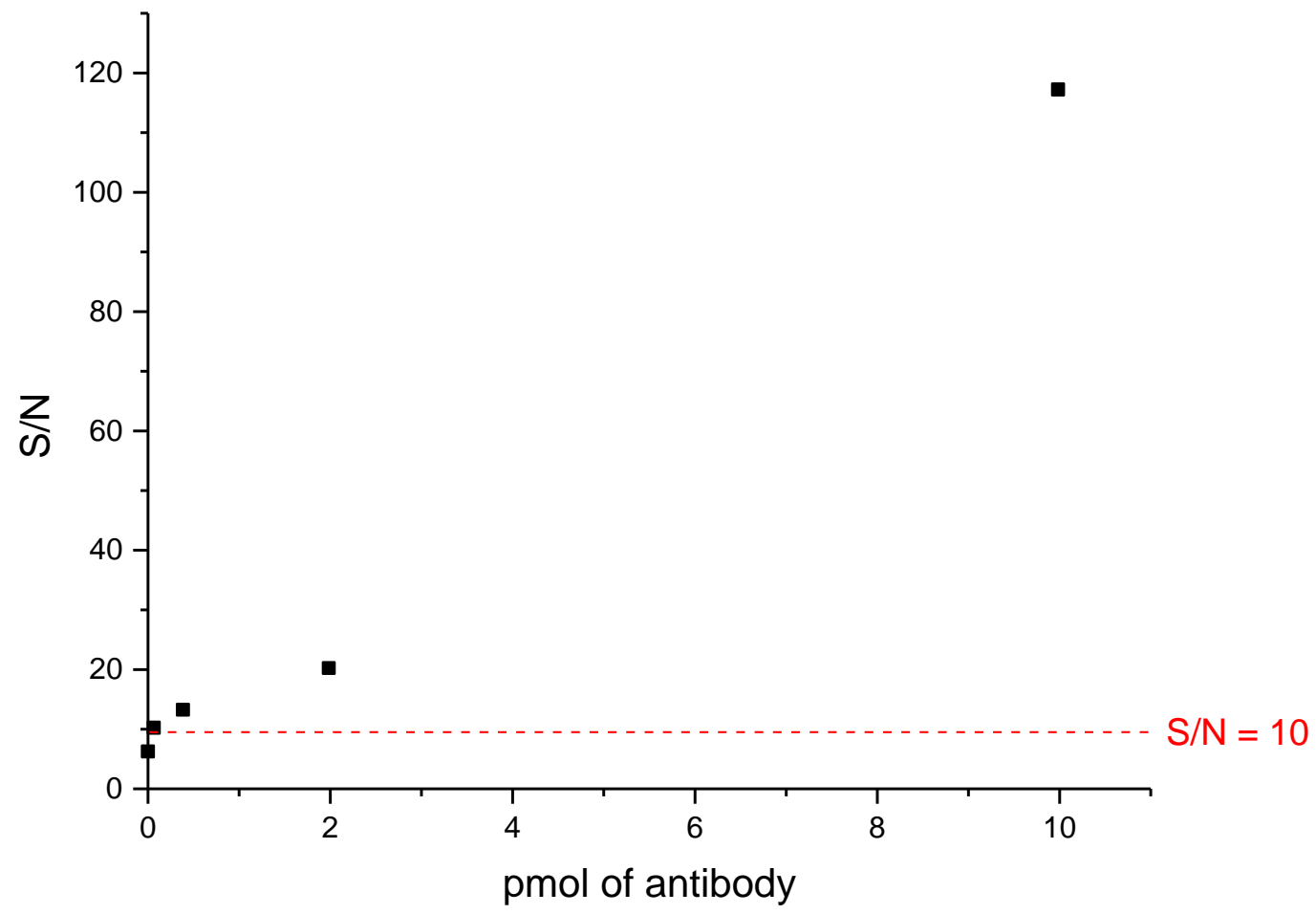

Supplemental Figure 4. Dependency of antigen signal in MALDI-TOF experiment after the in-situ enrichment on the amount of softlanded antibody. The graph shows that the capacity to bind antigen strongly depends on the amount of the antibody immobilized on the MALDI plate (system leptin/antileptin antibody). The $\mathrm{S} / \mathrm{N}=10$ level (as calculated by FlexAnalysis software) was found at $80 \mathrm{fmol}$ of soft-landed antibody. 